UFIFT-HEP-92-7

March, 1992

\title{
A Tumbling Top-Quark Condensate Model
}

\author{
Stephen P. Martin \\ Department of Physics \\ University of Florida \\ Gainesville, FL 32611
}

\begin{abstract}
We propose a renormalizable model with no fundamental scalars which breaks itself in the manner of a "tumbling" gauge theory down to the standard model with a top-quark condensate. Because of anomaly cancellation requirements, this model contains two color sextet fermions (quixes), which are vector-like with respect to the standard model gauge group. The model also has a large number of pseudo-Nambu-Goldstone bosons, some of which can be light. The top-quark condensate is responsible for breaking the electroweak gauge symmetry and gives the top quark a large mass. We discuss the qualitative features and instructive shortcomings of the model in its present form. We also show that this model can be naturally embedded into an aesthetically pleasing model in which the standard model fermion families appear symmetrically.
\end{abstract}


Recently there has been a great deal of interest in the idea[1-4] that the electroweak symmetry of the standard model is broken by a top-quark condensate. This would give a natural explanation for the fact that the top quark has a much larger mass than any of the other quarks and leptons, while simultaneously providing an electroweak symmetry breaking mechanism without a fundamental Higgs scalar.

In early versions of this idea, the top-quark condensate was supposed to be induced by a gauge-invariant but non-renormalizable four-fermion interaction

$$
\mathcal{L}_{\text {eff }} \sim \frac{g^{2}}{M^{2}}\left(\bar{Q}^{i} t\right)\left(\bar{t} Q_{i}\right)
$$

introduced at a scale $M$ which must be larger than the electroweak breaking scale. [Here $Q^{i}$ is the left-handed third generation quark doublet, the $i$ is an $S U(2)_{L}$ index, and $t$ is the right-handed part of the top quark. Color indices are suppressed.] If the coupling $g$ is large enough at the scale $M$, then a Nambu-Jona-Lasinio (NJL) mechanism[5] will trigger the formation of a top-quark vacuum expectation value ("condensate")

$$
\left\langle\bar{Q}^{i} t\right\rangle=\mu^{3} \delta^{i 1}
$$

which breaks the electroweak symmetry. The Higgs scalar boson is a composite top-antitop state bound by the interaction (1). The top-quark has a Yukawa coupling to the composite Higgs which is of order unity, and thus the top quark obtains a large mass.

The original formulation of the top-quark condensate idea is rather unsatisfying because it involves the $a d$ hoc and non-renormalizable interaction (1). Now, there is an obvious precedent for four-fermion interactions in elementary particle physics. The weak interactions were originally described by an effective four-fermion interaction which was later found to follow from integrating out massive intermediate vector gauge bosons. Similarly, one can imagine that (1) is the result of integrating out some heavy vector gauge bosons. In this scenario, the top quark is heavy because it couples to a new gauge interaction which is strongly coupled and spontaneously broken at a mass scale larger than the electroweak symmetry breaking scale. The most obvious way that this can happen is for the new interaction to be an asymptotically free non-abelian gauge theory. Then the 
running gauge coupling constant will increase as we go to lower mass scales. Eventually, the gauge coupling becomes large enough to drive the formation of condensates, and the new gauge symmetry is then spontaneously broken (e.g. by a mechanism to be proposed below) so that it does not confine. Several authors [6-12] have recently enumerated some possibilities for the form of the renormalizable theory. Other interesting extensions of and observations on the top-quark condensate idea are found in [13-26].

There are several important constraints on the top-quark condensate scenario which come from demanding that it arise from a renormalizable Lagrangian featuring a new nonabelian gauge interaction. These follow from the simple observation that if the top-quark has a special new gauge interaction, then other fermions must also have that gauge interaction in order for the full theory to be free of all gauge anomalies. Generally, these fermions will be "exotic", that is, they have transformation properties under the standard model gauge group which are different from the known quarks and leptons. Of course, the prediction of new exotic fermions from the top-quark condensate idea may be interesting if they are sufficiently heavy to have avoided discovery until now, but not heavy enough to avoid discovery forever. This can happen if the exotic fermions are in a complex representation of the full gauge group including the new strongly coupled interaction, but transform under the standard model subgroup as a real representation, so that they are eligible to receive masses. Note that one danger to be avoided in top-quark condensate model-building is that a priori these fermions might also participate in condensates which could break the standard model gauge group in unacceptable ways.

The new strongly coupled gauge interaction will have an approximate chiral symmetry which is spontaneously broken and includes the electroweak symmetry as a subgroup. Then, as in technicolor models, there will be a number of potentially light pseudo-NambuGoldstone bosons (PNGBs) which are bound states of the fermions which couple to the strong gauge interaction. These also may provide a means of experimental verification or falsification of any particular model. The specific properties of the extra fermions and PNGBs of course depend on the particular model, but models like the one we are going to consider here are always going to predict some non-standard-model phenomena of this 
kind. Traditionally, the economy of the top-quark condensate idea based on the nonrenormalizable interaction (1) has been used[4] to make predictions involving constraints on the top-quark and Higgs masses. In contrast, the non-economy implied by demanding renormalizability could provide a different kind of prediction involving the existence of non-standard model particles.

To build a renormalizable top-quark condensate model, one may select a gauge group $G$ which contains as a subgroup the standard model gauge group $G_{S M}=S U(3)_{c} \times S U(2)_{L} \times$ $U(1)_{Y}$. The fermions transform as an anomaly-free, complex representation of $G$. This representation contains the usual standard model quarks and leptons transforming in the usual way under $G_{S M}$, as well as some "extra" fermions which transform as a real represention of $G_{S M}$. A simple subgroup $H$ of $G$ becomes strongly coupled in the infrared, producing the top-quark condensate and possibly other condensates involving the other fermions which couple to $H$. Now, one must also have the spontaneous symmetry breaking $G \rightarrow G_{S M}$. Thus we are in a curious position: having explained the cause of electroweak symmetry breaking $G_{S M} \rightarrow G_{0}=S U(3)_{c} \times U(1)_{E M}$ by means of a top-quark condensate, we must now explain the origin of the symmetry breaking $G \rightarrow G_{S M}$ ! The purpose of this paper is to propose a renormalizable top-quark condensate model in which the spontaneous symmetry breaking occurs naturally without any fundamental scalar fields. This is accomplished by arranging that one of the "other" condensates breaks $G \rightarrow G_{S M}$ while the top-quark condensate breaks the electroweak symmetry. In other words, the theory with gauge group $G$ and an appropriate fermion representation automatically will break itself in the pattern $G \rightarrow G_{S M} \rightarrow G_{0}$, exactly in the manner of "tumbling gauge theories" [27]. Indeed, we will find it most convenient to employ the language and dynamical assumptions of [27] in order to get a qualitative understanding of our model.

We choose as a gauge group $G=S U(3)_{1} \times S U(3)_{2} \times S U(2)_{L} \times U(1)_{Y}$. The standard model color $S U(3)_{c}$ is the diagonal subgroup of $S U(3)_{1} \times S U(3)_{2}$. This is exactly the gauge structure used in Hill's recent "Topcolor" model[6]. ${ }^{\dagger}$ However, we choose a different

$\dagger$ This gauge group was also earlier employed in models[28] which have nothing to do with the top-quark condensate idea. 
set of fermion assignments for three reasons. First, the spontaneous symmetry breaking $G \rightarrow G_{S M}$ will be an automatic consequence of the condensation pattern given our choice of fermion representations, whereas [6] requires a fundamental scalar (or some unspecified dynamical mechanism) in order to provide this breaking. Second, as discussed in [12], the fermion representations in [6] contain a real representation of the unbroken gauge group $G$. This means that there are allowed bare mass terms in the case of [6] (one of which involves the right-handed part of the bottom quark) even before symmetry breaking. In order for [6] to work, one must make the assumption that those mass terms are prohibited by an ungauged global symmetry whose raison d'etre remains mysterious. Third, we will show at the end of this paper that our choice of fermion representations allows a natural extension to an aesthetically pleasing model which treats the three families of quarks and leptons in a symmetrical way.

We assign fermions to the following representations of $G=S U(3)_{1} \times S U(3)_{2} \times S U(2)_{L} \times$ $U(1)_{Y}$ :

$$
\begin{aligned}
& q_{1} \sim(\overline{\mathbf{6}}, \mathbf{1}, \mathbf{1},-1 / 3) \quad Q_{1}^{i}, Q_{2}^{i} \sim 2 \times(\mathbf{1}, \mathbf{3}, \mathbf{2}, 1 / 6) \\
& f_{1}, f_{2} \sim 2 \times(\mathbf{3}, \mathbf{3}, \mathbf{1}, 1 / 3) \quad c, u \quad \sim 2 \times(\mathbf{1}, \overline{\mathbf{3}}, \mathbf{1},-2 / 3) \\
& Q^{i} \sim(\mathbf{3}, \mathbf{1}, \mathbf{2}, 1 / 6) \quad d \quad \sim(\mathbf{1}, \overline{\mathbf{3}}, \mathbf{1}, 1 / 3) \\
& t \sim(\overline{\mathbf{3}}, \mathbf{1}, \mathbf{1},-2 / 3) \quad L_{1}^{i}, L_{2}^{i}, L_{3}^{i} \sim 3 \times(\mathbf{1}, \mathbf{1}, \mathbf{2},-1 / 2) \\
& q_{2} \quad \sim \quad(\mathbf{1}, \overline{\mathbf{6}}, \mathbf{1},-1 / 3) \quad \tau, \mu, e \quad \sim 3 \times(\mathbf{1}, \mathbf{1}, \mathbf{1}, 1)
\end{aligned}
$$

[The gauge transformation properties of fermions are always given in terms of left-handed two-component Weyl fields in (3) and throughout the rest of this paper.] It is easy to check that all of the gauge anomalies cancel with this fermion content.

How do the standard model fermions fit into (3)? After the symmetry breaking $S U(3)_{1} \times S U(3)_{2} \rightarrow S U(3)_{c}$, a fermion which transformed under $S U(3)_{1}$ as $\mathbf{R}_{1}$ and under $S U(3)_{2}$ as $\mathbf{R}_{\mathbf{2}}$ will transform under the diagonal $S U(3)_{c}$ as the direct product representation $\mathbf{R}_{\mathbf{1}} \times \mathbf{R}_{\mathbf{2}}$. So $f_{1}$ and $f_{2}$ each transform under $G_{S M}$ as $(\overline{\mathbf{3}}, \mathbf{1}, 1 / 3)+(\mathbf{6}, \mathbf{1}, 1 / 3)$. The two copies of $(\overline{\mathbf{3}}, \mathbf{1}, 1 / 3)$ in $f_{1}$ and $f_{2}$ are identified as the charge conjugates of two of the right-handed down-type quarks of the standard model. It is easy to see that (3) contains 
three standard model fermion families transforming under $G_{S M}$ as

$$
3 \times[(\mathbf{3}, \mathbf{2}, 1 / 6)+(\overline{\mathbf{3}}, \mathbf{1},-2 / 3)+(\overline{\mathbf{3}}, \mathbf{1}, 1 / 3)+(\mathbf{1}, \mathbf{2},-1 / 2)+(\mathbf{1}, \mathbf{1}, 1)]
$$

along with two vector-like quixes

$$
2 \times[(\mathbf{6}, \mathbf{1}, 1 / 3)+(\overline{\mathbf{6}}, \mathbf{1},-1 / 3)] \quad .
$$

Note that the quixes are in a real representation of the standard model gauge group and are thus eligible to receive masses after the symmetry breaking $G \rightarrow G_{S M}$. Also note that fractional electric charges are confined in this model.

In order to understand the symmetry breaking and generation of masses in this model, let us now suppose that all of the gauge couplings are weak at some sufficiently high energy scale and consider what happens as we move to lower energy scales. Note that $S U(3)_{1}$ and $S U(3)_{2}$ are both asymptotically free; their $\beta$-functions are given to one loop order by

$$
\begin{aligned}
\beta_{1} & =\mu \frac{d g_{1}}{d \mu}=-\frac{19}{48 \pi^{2}} g_{1}^{3} \\
\text { and } \quad \beta_{2} & =\mu \frac{d g_{2}}{d \mu}=-\frac{15}{48 \pi^{2}} g_{2}^{3}
\end{aligned}
$$

Therefore it is quite reasonable to assume that $S U(3)_{1}$ becomes strongly coupled first in the infrared, while the other couplings remain small. Thus $S U(3)_{1}$ plays the role of $H$ in this model.

In order to understand the pattern of fermion condensation in our model, we may turn to the dynamical assumptions outlined in [27], which we now briefly review. Consider a model which consists of an asymptotically free gauge theory which couples to some fermions but no scalars. The fermions may also have weakly coupled gauge interactions whose effects may be treated perturbatively. When the strong gauge coupling becomes sufficiently large in the infrared, a scalar fermion bilinear condensate will form in an irreducible representation of the gauge group. Suppose that the fermions involved in the condensate transform under the strongly coupled gauge group in the irreducible representations $R_{1}$ and $R_{2}$, and the resulting condensate transforms as $R_{s}$. (We treat all the fermions here 
as left-handed two-component Weyl fermions.) Thus $R_{s}$ occurs in the direct sum decomposition of the direct product $R_{1} \times R_{2}=R_{s}+\cdots$. We need a way of deciding for which choices of $R_{1}, R_{2}$, and $R_{s}$ the condensate will occur. According to the single gauge boson exchange approximation, the condensate appears in the "most attractive scalar channel" (MASC), $R_{1} \times R_{2} \rightarrow R_{s}$, for which $V=C_{1}+C_{2}-C_{s}$ is largest. Here $C_{1}, C_{2}$, and $C_{s}$ are the quadratic Casimir invariants for the representations $R_{1}, R_{2}$, and $R_{s}$, respectively. [For example, if the strongly coupled interaction were a $U(1)$, and left-handed fermions had charges $q_{1}$ and $q_{2}$, then $V \propto q_{1}^{2}+q_{2}^{2}-\left(q_{1}+q_{2}\right)^{2}=-2 q_{1} q_{2}$, so that for a collection of charged fermions, the most attractive channel occurs when the product of charges is most negative. Thus in a general gauge theory the statement that $V$ should be maximized is the generalization of the familiar statement in electrodynamics that opposite charges attract.] The fermions which participate in the condensate obtain masses at this stage, as do the gauge bosons corresponding to those generators of the gauge symmetry which are spontaneously broken by the condensate. The remaining gauge bosons and fermions define the next stage of the tumbling.

In the case of our model, the strongly coupled $S U(3)_{1}$ has LH fermions transforming as a $\overline{\mathbf{6}}$, eight $\mathbf{3}$ 's, and one $\overline{\mathbf{3}}$. The most attractive channels for this fermion content, and their relative strengths $V$, are as follows:

$$
\begin{aligned}
& \text { Channel } \quad \underline{V} \\
& \overline{\mathbf{6}} \times \mathbf{3} \rightarrow \overline{\mathbf{3}} \quad 10 \\
& \overline{\mathbf{6}} \times \overline{\mathbf{6}} \rightarrow \mathbf{6} \quad 10 \\
& \mathbf{3} \times \overline{\mathbf{3}} \rightarrow \mathbf{1} \quad 8 \\
& \overline{\mathbf{6}} \times \overline{\mathbf{3}} \rightarrow \mathbf{8} \quad 5 \\
& \mathbf{3} \times \mathbf{3} \rightarrow \overline{\mathbf{3}} \quad 4 \quad \text {. }
\end{aligned}
$$

From (8) we see that the most naive version of the tumbling hypothesis is ambiguous, since there is a tie for the MASC between the channels $\overline{\mathbf{6}} \times \mathbf{3} \rightarrow \overline{\mathbf{3}}$ and $\overline{\mathbf{6}} \times \overline{\mathbf{6}} \rightarrow \mathbf{6}$. We must decide which of these condensates actually forms in order to proceed.

Fortunately, other authors[29-30] have already worried about what happens when there 
is such a tie for the MASC in a tumbling gauge theory. According to their criteria, the winner in our model is the channel $\overline{\mathbf{6}} \times \mathbf{3} \rightarrow \overline{\mathbf{3}}$. More specifically, according to the arguments of [30] and [31], the condensate forms according to

$$
\left\langle q_{1}^{(\alpha \beta)} f_{1 \gamma a}\right\rangle=M^{3} \delta_{\gamma}^{(\alpha} \delta_{a}^{\beta)}
$$

(We use Greek letter $\alpha, \beta \ldots$ and Latin letters $a, b \ldots$ for indices in the fundamental representations of $S U(3)_{1}$ and $S U(3)_{2}$ respectively.) The composite scalar field $\Phi_{a}^{\alpha}=q_{1}^{(\alpha \beta)} f_{1 \beta a}$ transforms under $G$ as $(\overline{\mathbf{3}}, \mathbf{3}, \mathbf{1}, 0)$ and obtains a $\operatorname{VEV}\left\langle\Phi_{a}^{\alpha}\right\rangle=2 M^{3} \delta_{a}^{\alpha}$. This condensate breaks $S U(3)_{1} \times S U(3)_{2} \rightarrow S U(3)_{c}$ as promised. Equation (9) reflects not only the assumed preference of the strongly coupled theory for the channel $\overline{\mathbf{6}} \times \mathbf{3} \rightarrow \overline{\mathbf{3}}$, but also the solution to a vacuum alignment problem[31], namely which of the eight 3's of $S U(3)_{1}$ will condense with $q_{1}$. There is a simple heuristic reason why the condensate chooses to leave $S U(3)_{c}$ unbroken as in (9); this is because the fermions participating in the condensate (9) transform as a $\mathbf{6}$ and a $\overline{\mathbf{6}}$ of $S U(3)_{c}$ and thus feel an additional attractive force which would not be present if the $q_{1}$ chose to condense in such a way as to break $S U(3)_{c}$. (Of course, the choice of $f_{1}$ instead of $f_{2}$ in (9) is completely arbitrary.)

Of the sixteen gauge bosons associated with $S U(3)_{1} \times S U(3)_{2}$, eight remain massless after the symmetry breaking and are the gluons of QCD. The other eight gauge bosons obtain a mass of order $g_{1} M$ and also transform as an octet of $S U(3)_{c}$. If one integrates out these heavy gauge bosons, one obtains[6] precisely the four-fermion interaction (1) which was our original motivation (along with some weaker four-fermion interactions). If the coupling constants of $S U(3)_{1}$ and $S U(3)_{2}$ at the scale $M$ are $g_{1}$ and $g_{2}$, then it is easy to show that the QCD coupling constant at $M$ is given by $g_{c}=g_{1} g_{2} / \sqrt{g_{1}^{2}+g_{2}^{2}}$. We assume that $S U(3)_{1}$ is strongly coupled at $M$ and $S U(3)_{2}$ is not, so that $g_{1} \gg g_{2}$ and $g_{c} \approx g_{2}$.

According to (9), all of the components of $q_{1}$ condense, along with the part of $f_{1}$ which transforms as a 6 of $S U(3)_{c}$. This quix receives a mass and decouples from the tumbling. Another quix, consisting of $q_{2}$ and the part of $f_{2}$ which transforms as a $\mathbf{6}$ of $S U(3)_{c}$, remains uncondensed and massless at this stage. The uncondensed parts of $f_{1}$ and $f_{2}$ which transform as $\overline{3}$ 's of $S U(3)_{c}$ are the charge conjugates of two of the right-handed 
down-type quarks of the standard model and remain massless at this stage.

The next most attractive scalar channel in (8) (not including the channel $\overline{\mathbf{6}} \times \overline{\mathbf{6}} \rightarrow \mathbf{6}$, because the $\overline{\mathbf{6}}$ has already condensed) is the $\mathbf{3} \times \overline{\mathbf{3}} \rightarrow \mathbf{1}$. Since the strength of the attraction in this channel is only slightly less than that of the MASC, we make the dynamical assumption that this condensate is also triggered even though (9) breaks $S U(3)_{1}$. In fact, this corresponds to the assumption in the NJL language that the four-fermion interaction (1) is sufficiently attractive to produce a top-quark condensate. Now there is again a vacuum alignment problem since the $\overline{\mathbf{3}}$ has a choice of 3's with which to condense. Again, the condensate will choose to avoid breaking $S U(3)_{c}$, so that the condensate is of the form

$$
\left\langle Q_{\alpha}^{i} t^{\beta}\right\rangle=\mu^{3} \delta^{i 1} \delta_{\alpha}^{\beta}
$$

This is just the top-quark condensate which was our original motivation, with color indices restored. Heuristically, the theory prefers (10) because $Q^{i}$ and $t$ transform as a $\mathbf{3}$ and $\overline{\mathbf{3}}$ of $S U(3)_{c}$, and this provides an additional attractive force which would not be present for any other condensate which breaks $S U(3)_{c}$. Of course, the $\delta^{i 1}$ is just an arbitrary choice of orientation of weak isospin. The condensate (10) breaks $S U(2)_{L} \times U(1)_{Y} \rightarrow U(1)_{E M}$ with the composite field $\phi^{i}=Q_{\alpha}^{i} t^{\alpha}$ playing the role of the standard model Higgs scalar boson. The top quark condenses and gets a mass, as do the $W^{ \pm}$and $Z^{0}$ vector bosons.

As we move further into the infrared, the next interesting thing that happens is that the remaining light quix condenses, due primarily to the QCD force, and so obtains a large constituent mass. This condensate has the form

$$
\left\langle q_{2}^{(a b)} f_{2 \alpha c}\right\rangle=m^{3} \delta_{\alpha}^{(a} \delta_{c}^{b)}
$$

This condensate can occur at a much higher energy scale than for the ordinary quarks in QCD, because the quadratic Casimir invariant of the 6 of $S U(3)$ is $5 / 2$ times that of the 3. The constituent mass of the lighter quix could therefore be as high as a few hundred GeV. (This fact has been exploited by Marciano[32] who suggested that a quix condensate driven by QCD could be responsible for electroweak symmetry breaking. The quix in our model plays a quite different role, since it is an $S U(2)_{L}$ singlet and our quix condensates 
do not break $\left.G_{S M}.\right)$ The lighter quix can also get a mass which is a current mass from the point of view of the standard model interactions, by integrating out the heavy octet of gauge bosons, and by mixing with the heavier quix due to some additional interactions at higher energies.

A quix will be pair-produced at hadron colliders by gluon fusion. In our model, each quix will decay by emitting a heavy color octet vector boson, turning into one of the downtype quarks which are components of $f_{1}$ and $f_{2}$. The heavy vector boson will then decay into a quark-anti-quark pair. Thus the experimental signature for the quix should consist of a six-jet signal above the QCD background. For a quix with mass in the hundred GeV range, such a signal is difficult but not impossible to detect at the Tevatron, LHC, or $\mathrm{SSC}[33]$.

Let us now consider the spectrum of PNGBs which arise from our model. The $S U(3)_{1}$ interaction has an approximate chiral symmetry $S U(3)_{1} \times S U(8) \times U(1) \times U(1)$. (There would be three $U(1)$ 's, but one of them has an $S U(3)_{1}$ anomaly.) The first condensate (9) breaks this down to $S U(3)_{c} \times S U(2)_{L} \times U(1)_{Y} \times U(1) \times U(1)$. There are therefore 59 PNGBs from this stage, of which 8 are eaten and give mass to the $S U(3)_{c}$ octet of heavy gauge bosons. The second condensate (10) further breaks the chiral symmetry down to $S U(3)_{c} \times U(1)_{E M} \times U(1) \times U(1)$. There are three would-be Nambu-Goldstone bosons from this stage which are eaten by the $W^{ \pm}$and $Z^{0}$ vector bosons. Finally, the lighter quix condensate (11) breaks an additional $U(1)$, so that the original chiral symmetry is broken down to $S U(3)_{C} \times U(1)_{E M} \times U(1)_{B}$. The baryon number $U(1)_{B}$ is an exactly conserved, non-anomalous global symmetry of the $S U(3)_{1} \times S U(3)_{2}$ interactions (but has the usual $S U(2)_{L}$ anomaly of the standard model). So there are 52 uneaten PNGBs. Of these, 24 transform as eight 3's and 24 more transform as three 8's of $S U(3)_{c}$. These colored PNGBs obtain large masses as in technicolor models. However, the remaining four axionlike neutral PNGBs may be dangerously light.

The model we have described here clearly cannot be complete as it stands. Perhaps the most glaring evidence of this is that the leptons remain massless and in fact are decoupled 
from the symmetry breaking sector. One might imagine that the lighter quarks and leptons can be given realistic masses by adding in higher order interactions analogous to those in extended technicolor models. Such interactions might have the additional beneficial effect of contributing to the masses of the neutral PNGBs mentioned in the previous paragraph. Of course, one may also expect to encounter the same problems that occur in extended technicolor. For example, the required additional interactions may give rise to flavor changing neutral current interactions at an unacceptable level. The most obvious way to try to couple the leptons to the symmetry breaking sector is to embed $S U(3)_{1}$ into a Pati-Salam $S U(4)$ at some high energy scale. There are several inequivalent ways to embed the fermion content (3) into the enlarged gauge group; so far we have not found any particularly satisfying way to do it.

Another potential disaster for our model involves the parameter $\rho=M_{W}^{2} / M_{Z}^{2} \cos ^{2} \theta_{W}$, which is constrained experimentally to be very near 1 . The usual way of ensuring this in dynamical electroweak symmetry breaking models, as in the standard model with a fundamental Higgs, is to arrange for a "custodial" $S U(2)$ symmetry[34] of both the Lagrangian and the vacuum, under which the generators of $S U(2)_{L}$ transform as a triplet. Our model has no such custodial $S U(2)$. However, the situation may not be completely hopeless; consider for example the scenario of [4] in which $M$ is taken to be $\gg 246 \mathrm{GeV}$. The effective theory far below $M$ looks like the standard model with a heavy top quark and a Higgs doublet, so that if the top quark is arranged to not be too heavy, the $\rho$-parameter could come out in the allowed range. Now, the renormalization group methods used in [4] rely for their validity on the assumption that the theory is already fine-tuned, so that the scale of new physics is much larger than the electroweak scale. Since the avoidance of fine-tuning is one of the main motivations for investigating dynamical electroweak symmetry breaking in the first place, we tend to favor the opposite possibility, namely that the symmetry breaking $G \rightarrow G_{S M}$ occurs at a scale not too far removed from the scale of electroweak symmetry breaking. In this case, the arguments used in [4] are not reliable and should not be used to draw quantitative conclusions; in particular the prediction of a very heavy top might be avoided. If the scale $M$ is sufficiently close to $246 \mathrm{GeV}$, there will certainly be no 
range of energy scales at which the interaction (1) alone comes close to accurately reflecting the strong coupling dynamics. Furthermore, the effective theory will contain a much more complicated spectrum of composite resonances than just a Higgs doublet. These resonances are also bound states of the fermions which couple to $S U(3)_{1}$. There should be, for example, composite vector particles exactly analogous to the techni- $\rho$ and techni- $\omega$ of technicolor models. (This has been emphasized already in [26].) Perhaps some presently mysterious feature of the strong coupling dynamics prefers $\rho \approx 1$. Or perhaps the value of $M$ is small enough to invalidate the quantitative conclusions of [4] without invalidating the qualitative conclusion that the effective theory below $M$ consists of the standard model with one Higgs doublet and other resonances and interactions which violate the custodial $S U(2)$ in a controlled way. To analyze whether these (perhaps optimistic) possibilities can be realized requires an improved understanding of the rather murky dynamics of strongly coupled spontaneously broken theories, especially since the condensates (9) and (10) have close to the same strength in the single gauge boson approximation.

The model we have presented here can be embedded into a very symmetric-looking model by introducing another gauged $S U(3)$. Thus we now take the unbroken gauge group to be $G^{\prime}=S U(3)_{1} \times S U(3)_{2} \times S U(3)_{3} \times S U(2)_{L} \times U(1)_{Y}$, and we take the fermions to transform as:

$$
\begin{aligned}
q_{1}, q_{2}, q_{3} & \sim(\overline{\mathbf{6}}, \mathbf{1}, \mathbf{1}, \mathbf{1},-1 / 3)+(\mathbf{1}, \overline{\mathbf{6}}, \mathbf{1}, \mathbf{1},-1 / 3)+(\mathbf{1}, \mathbf{1}, \overline{\mathbf{6}}, \mathbf{1},-1 / 3) \\
f_{1}, f_{2}, f_{3} & \sim(\mathbf{3}, \mathbf{3}, \mathbf{1}, \mathbf{1}, 1 / 3)+(\mathbf{3}, \mathbf{1}, \mathbf{3}, \mathbf{1}, 1 / 3)+(\mathbf{1}, \mathbf{3}, \mathbf{3}, \mathbf{1}, 1 / 3) \\
Q_{3}^{i}, Q_{1}^{i}, Q_{2}^{i} & \sim(\mathbf{3}, \mathbf{1}, \mathbf{1}, \mathbf{2}, 1 / 6)+(\mathbf{1}, \mathbf{3}, \mathbf{1}, \mathbf{2}, 1 / 6)+(\mathbf{1}, \mathbf{1}, \mathbf{3}, \mathbf{2}, 1 / 6) \\
t, c, u & \sim(\overline{\mathbf{3}}, \mathbf{1}, \mathbf{1}, \mathbf{1},-2 / 3)+(\mathbf{1}, \overline{\mathbf{3}}, \mathbf{1}, \mathbf{1},-2 / 3)+(\mathbf{1}, \mathbf{1}, \overline{\mathbf{3}}, \mathbf{1},-2 / 3) \\
L_{1}^{i}, L_{2}^{i}, L_{3}^{i} & \sim 3 \times(\mathbf{1}, \mathbf{1}, \mathbf{1}, \mathbf{2},-1 / 2) \\
\tau, \mu, e & \sim 3 \times(\mathbf{1}, \mathbf{1}, \mathbf{1}, \mathbf{1}, 1)
\end{aligned}
$$

Note that the fermion content is now invariant under interchange of the three $S U(3)$ 's. Furthermore, the colored fermions are arranged in irreducible representations which each occur only once. By analogy with the "Topcolor" of [6] and the "Chiral Color" of [28], it is tempting to refer to this enlarged model as "Family Color", since the three $S U(3)$ 
interactions in $G^{\prime}$ are associated with the three families. In order to recover our previous model, we just assume that $S U(3)_{2} \times S U(3)_{3}$ breaks down to the diagonal $S U(3)$ subgroup, which is identified with the $S U(3)_{2}$ of $G$. [It is suggestive that such a breakdown would be caused by a condensation of the $(\mathbf{1}, \mathbf{1}, \overline{\mathbf{6}}, \mathbf{1},-1 / 3)$ with the $(\mathbf{1}, \mathbf{3}, \mathbf{3}, \mathbf{1}, 1 / 3)$ exactly analogous to the condensate $(9)$, if $S U(3)_{3}$ gets strong at a very high scale. However, we think it prudent to refrain from extending our dynamical assumptions too far beyond the realm of the standard model, since as we have already noted, we are missing (at least) some major ingredient which is responsible for generating lepton masses.] The remaining unbroken gauge group is then $G$, and the fermion content is precisely that of eq. (3) plus a quix which is vector-like with respect to the gauge group $G$ and therefore presumeably gets a large mass at this stage. We find it encouraging that the somewhat haphazard-looking fermion content given in (3) actually can come from the more attractive (12). This is of course just one of the possible extensions of the basic model with gauge group $G$ and fermion content (3).

In this paper, we have described a model for dynamical electroweak symmetry breaking which borrows from the old idea of tumbling gauge theories and the younger top-quark condensate idea. We have not attempted to draw any precise quantitative conclusions, being content with the qualitative observation that the gauge symmetries are broken in the correct way and that the top quark obtains a large mass. In any case, we need additional model-building ideas in order to have a chance for a realistic mass spectrum for the lighter quarks and leptons, and additional technical ideas in order to calculate reliably without fine-tuning in the strongly coupled theory. The model we have discussed here is an example of a dynamical electroweak symmetry breaking scheme which is similar to the technicolor idea, with the top quark playing the role of a techniquark, but differs in that the strongly coupled gauge theory is broken instead of confining in the infrared. Note that there are, qualitatively speaking, three possible fates for an asymptotically free non-abelian gauge theory in the infrared. The first possibility is that the theory can become spontaneously broken before it has a chance to become strong; this is the fate of $S U(2)_{L}$ in the standard model. The second possibility is that the theory can become 
strongly coupled and confining without being broken; we understand this because it is what happens to QCD in the standard model. It is also what is supposed to happen in technicolor theories. The third possibility is that the theory can become strong enough to produce condensates, but is then spontaneously broken so that it does not confine. There is no standard model example of this, but there is also no good reason why such a thing could not happen between the electroweak scale and the Planck scale. Despite its shortcomings in the present incarnation, we hope that our model illustrates how this third possibility could be responsible for breaking the electroweak symmetry.

I am grateful to Jonathan Bagger, Paul Griffin, Chris Hill, Pierre Ramond, Dave Robertson and Pierre Sikivie for helpful comments. This work was supported in part by the Institute for Fundamental Theory and by DOE contract DE-FG05-86-ER40272.

\section{References}

1. Y. Nambu, EFI Report No. 88-39, 1988 (unpublished); in New Trends in Strong Coupling Gauge Theories, 1988 International Workshop, Nagoya, Japan, edited by M. Bando, T. Muta, and K. Yamawaki), (World Scientific, Singapore, 1989); EFI Report No. 89-08, 1989 (unpublished).

2. V. Miransky, M. Tanabashi, and K. Yamawaki, Phys. Lett. B221, 177, (1989); Mod. Phys. Lett. A 4, 1043, (1989).

3. W. J. Marciano, Phys. Rev. Lett. 62, 2793, (1989); Phys. Rev. D41, 219, (1990).

4. W. A. Bardeen, C. T. Hill, and M. Lindner, Phys. Rev. D41, 1647, (1990).

5. Y. Nambu and G. Jona-Lasinio, Phys. Rev. 122, 345, (1961).

6. C. T. Hill, Phys. Lett. B266, 419, (1991).

7. M. Lindner and D. Ross, Nucl. Phys. B370, 30, (1992).

8. D. E. Clague and G. Ross, Nucl. Phys. B364, 43, (1991). 
9. T. K. Kuo, U. Mahanta and G. T. Park Phys. Lett. B248, 119, (1990).

10. R. Bonisch, "Gauge Created Top Quark Condensate and Heavy Top", Munich University preprint LMU-91-03, August 1991.

11. S. F. King, Phys. Rev. D45, 990, (1992).

12. S. P. Martin, "Renormalizable Top-Quark Condensate Models", Florida preprint UFIFT-HEP-91-24, October 1991, to appear in Physical Review D.

13. T. E. Clark, S. T. Love, and W. A. Bardeen, Phys. Lett. B237, 235, (1990).

14. M. A. Luty, Phys. Rev. D41, 2893, (1990).

15. M. Suzuki, Phys. Rev. D41, 3457, (1990); Mod. Phys. Lett. A5, 1205, (1990).

16. P. Kaus and S. Meshkov, Phys. Rev. D42, 1863, (1990).

17. S. F. King and S. H. Mannan, Phys. Lett. B241, 249, (1990); Phys. Lett. B254, 197, (1991).

18. K. S. Babu and R. N. Mohapatra, Phys. Rev Lett. 66, 556, (1991).

19. F. Barrios, U. Mahanta, Phys. Rev. D43, 284, (1991).

20. M. Carena, T. Clark, C. Wagner, W. A. Bardeen, and K. Sasaki, "Dynamical Symmetry Breaking and the Top-Quark Mass in the Minimal Supersymmetric Standard Model", preprint FERMILAB-PUB-91/96-T.

21. M. Bando, T. Kugo, N. Maekawa, N. Sasakura, Y. Watabiki, and K. Suehiro, Phys. Lett. B246, 466, (1990).

22. C. T. Hill, M. A. Luty, and E. A. Paschos, Phys. Rev. D43, 3011, (1991).

23. Y. Achiman and A. Davidson, Phys. Lett. B261, 431, (1991).

24. S. P. Martin, Phys. Rev. D44, 2892, (1991). 
25. A. Hasenfratz, P. Hasenfratz, K. Jansen, J. Kuti and Y. Shen, Nucl. Phys. B365, $79,(1991)$.

26. M. Lindner and D. Lüst, Phys. Lett. B272, 91, (1991).

27. S. Raby, S. Dimopolous, and L. Susskind, Nucl. Phys. B169, 373, (1980).

28. P. H. Frampton and S. L. Glashow, Phys. Lett. B190, 157, (1987); Phys. Rev. Lett. 58, 2168, (1987).

29. D. Amati and M. A. Virasoro, Phys. Lett. B99, 225, (1981).

30. V. P. Gusynin, V. A. Miransky and Yu. A. Sitenko, Phys. Lett. B123, 407, (1983).

31. S. Weinberg, Phys. Rev. D13, 974, (1976); M. Peskin, Nucl. Phys. B175, 197, (1980); J. Preskill, Nucl. Phys. B177, 21, (1981).

32. W. J. Marciano, Phys. Rev. D21, 2425, (1980).

33. R. S. Chivukula, M. Golden, and E. H. Simmons, Phys. Lett. 257B, 403, (1991); Nucl. Phys. B363, 83, (1991).

34. P. Sikivie, L. Susskind, M. Voloshin and V. Zakharov, Nucl. Phys. B173, 189, (1980). 